\title{
Research Paper: The Effect of TheraBand Training on the Q Angle and Distance of Ankle Medial Malleolus in Individuals With Genu Valgum Deformity
}

\author{
Shiva Bahadori' ${ }^{1}$, Hooman Fatahi $^{1^{*}}$ (D), Mansooreh Ahmadpoor ${ }^{1}$ (D) \\ 1. Department of Sports Injury and Corrective Exercises, Isfahan (Khorasgan) Branch, Islamic Azad University, Isfahan, Iran.
}

Citation Bahadori Sh, Fatahi H, Ahmadpoor M. The Effect of TheraBand Training on the Q Angle and Distance of Ankle Medial Malleolus in Individuals With Genu Valgum Deformity. Physical Treatments. 2020; 10(3):117-126. http://dx.doi. org/10.32598/ptj.10.3.304.3

http://dx.doi.org/10.32598/ptj.10.3.304.3

Keywords:

TheraBand, Q Angle, Deformity, Genu valgum

\section{A B S T RA C T}

Purpose: Genu valgum deformity a common lower extremity deformity. The weakness of the lower limb muscles followed by the internal rotation of the femur and external rotation of the tibia, shift the gravitational pull to the outside of the knee. This lower limb alignment deformity could increase the distance of ankle medial malleolus and the Q angle. Therefore, the present study aimed to investigate the effect of TheraBand training on the $\mathrm{Q}$ angle and distance of ankle medial malleolus in individuals with genu valgum deformity.

Methods: This was a quasi-experimental study with a pre-test and post-test and control group design. The study population consisted of girls in the age range of 9-13 years with genu valgum deformity. The study subjects were randomly divided into the experimental $(\mathrm{n}=15)$ and control $(n=15)$ groups. The experimental group participated in TheraBand exercises for 8 weeks and 3 sessions per week. However, the control group received no training during this period. Before and after 8 weeks, the $\mathrm{Q}$ angle and distance of ankle medial malleolus were measured by goniometers and coulis, respectively. Analysis of Covariance (ANCOVA) was used to analyze the collected data in SPSS. The significance level was considered at 0.05 .

Results: The obtained statistical results suggested that TheraBand training significantly decreased the $\mathrm{Q}$ angle and the distance of ankle medial malleolus in the explored subjects with genu valgum deformity ( $\mathrm{P}=0.001 \& \mathrm{P}=0.04$, respectively). However, no significant difference was observed in the control group.

Conclusion: The obtained data revealed that TheraBand training could improve the genu valgum position. Therefore, this training protocol could be implemented to improve the deformity in this population.

\footnotetext{
* Corresponding Author:

Hooman Fatahi, PhD.

Address: Department of Sports Injury and Corrective Exercises, Isfahan (Khorasgan) Branch, Islamic Azad University, Isfahan, Iran.

Phone: +98 (903) 2485812

E-mail: hoomanfatahi92@yahoo.com
} 


\section{Highlights}

- The TheraBanddd exercise improves the Q angle in individuals with genu valgum abnormality.

- The TheraBand exercise improve distance of ankle medial malleolus in individuals with genu valgum abnormality.

\section{Plain Language Summary}

Physical posture is the alignment of different body parts with each other. When an individual is in a poor physical posture, due to the high pressure on different body parts, the body direction generates a deformity. This constant pressure, even if relatively low, could cause non-anatomical compatibility. Genu valgum also increases the Q angle in this group. The Q angle is the angle between the quadriceps muscle line and the patellar tendon line, i.e. reported to be 10-14 degrees in men and 14-18 in women. TheraBandd exercises, as a safe tool, are effective to improve the neuromuscular system, muscle strength, and the ability to perform functional tasks. The obtained statistical results suggested that TheraBandd training significantly decreased the Q angle and the distance of ankle medial malleolus in the explored subjects with genu valgum deformity.

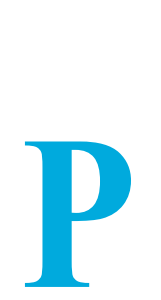

\section{Introduction}

hysical posture is the alignment of different body parts with each other. When an individual is in a poor physical posture, due to the high pressure on different body parts, the body direction generates a deformity; this constant pressure, even if relatively low, could cause non-anatomical compatibility. These changes alter individuals' ability to perform activities and affect the overall efficiency of the body [1].

The knee joint, like all other joints in the body, is affected by defects, injuries, and diseases. Moreover, unlike the shoulder joint, the elbow and wrist must bear the body weight while moving [2]. The most frequent deformities of the knee, due to the correctability and improvement in the range of corrective movements studied, include the genu varum, genu valgum, flat foot, hallux valgus, cavus foot, and hammertoe [3].

The genu varum may be caused by weakness in the lateral muscles, such as the Tensor fasciae latae, Biceps femoris, and Fibular muscles, as well as the shortness of the medial muscles, such as the Semimembranosus and Semitendinosus muscles, Gracilis, and Tibialis anterior [3]. In this deformity, when the individual is in standing posture with the ankle medial malleolus of the foot together, the knees remain completely separate [4]. According to previous studies, TheraBand could strengthen weak muscles; accordingly, it improves the direction of the femur, tibia, and ankles of individuals with genu varum [5]. One of the most prevalent deformities, i.e. more common in females, is genu valgum deformity
[6]. In this condition, the weight line [Center of Gravity (COG)] that descends from the center of the hip joint tends to the outside of the knee joint. Subsequently, it alters the pattern of body weight applied to the medial and lateral parts of the knee joint [7]. Genu valgum also increases the Q angle in this group. The Q angle is the angle between the quadriceps muscle line and the patellar tendon line, i.e. reported to be 10-14 degrees in men and 14-18 in women. This difference is due to the shape of the females' pelvic [4]. Wider pelvic, hip, and tibia rotation, and genu valgum deformity affect $\mathrm{Q}$ angle, and ultimately optimal posture [8]. Additionally, due to the changes in the location of the gravity line and the abnormal pressure on different parts of the joint surfaces, the knee will be prone to premature erosive changes; subsequently, early arthritis could be expected [9]. Advanced physiological valgus cases after the age of 8 years may lead to patellar bone misalignment and the instability of the knee ligaments. Such a condition lead to gait and running problems, as well as lower limb deformities [10].

Physical exercise, as a cheap, accessible, non-invasive, and low-risk approach in maintaining health and mobility or improving the alignment of the lower limbs are recognized [11]. TheraBand exercises are recently employed because they are more comfortable and safer. An advanced resistance exercise is implementing TheraBand, which could increase muscle size and strength in individuals [12]. TheraBand exercises, as a safe tool are effective to improve the nervous-muscular system, muscle strength, and the ability to perform functional tasks [11]. For approximately 25 years, researchers have been using TheraBand resistance exercises to increase strength and posture stability, and have achieved effec- 
tive results [13]. Elastic resistance (TheraBand) has been proven to increase strength, mobility, and function, as well as reduce joint pain. TheraBand is inexpensive, portable, and versatile. Javanmehr et al. (2018) also stated that muscle strength could be the most effective manner of preventing and treating deformities [14]. Park et al. (2017) demonstrated that TheraBand training and stretching exercises are effective methods to correct the genu varum [15]. Lovell et al. (2010) argued that highintensity strength training increases the strength and power of the elderly [16]. Delshad et al. (2011) stated that TheraBand strength training could prevent muscle disability in women [17]. Saki et al. (2019) documented that plyometric exercises in women with dynamic knee valgus could increase the strength of weakened muscles in them [18]. Resistance training with TheraBand provides strength; this resistance is created by stretching force and could have a strengthening effect on weakened muscles. Therefore, strengthening the weakened muscles in the lower limbs to reduce the angle $\mathrm{Q}$ and improve the genu valgum deformity is essential. Scholars disregarded investigating this training method and the effect of TheraBand on the genu valgum deformity. Thus, the present research aimed to investigate the effect of 8 weeks of TheraBand training on the Q angle and the distance of ankle medial malleolus in individuals with genu valgum deformity.

\section{Materials and Methods}

This was a quasi-experimental study with a pre-test, post-test and a control group design. The statistical population of the present study consisted of 13-9-year-old

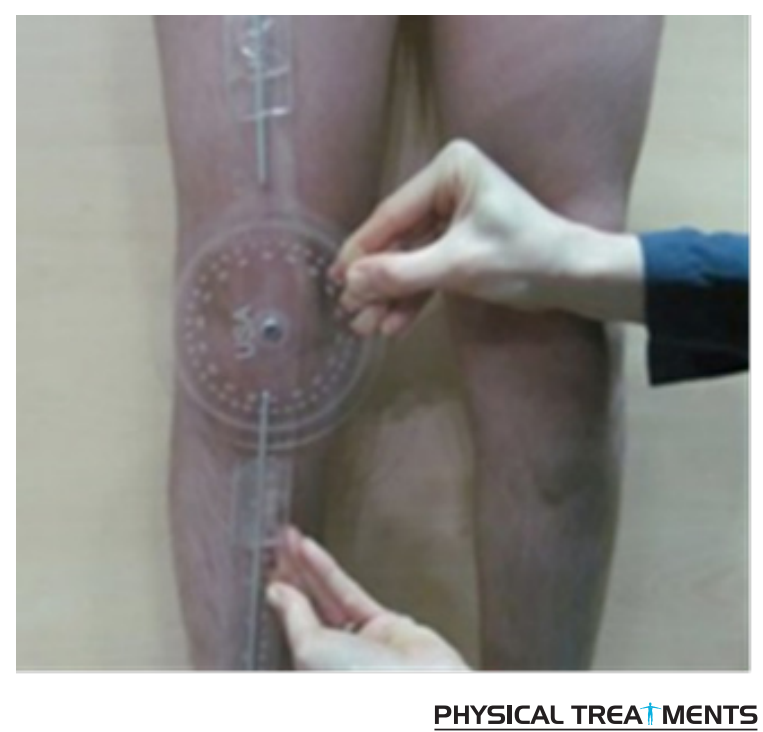

Figure 1. Measuring the angle $\mathrm{Q}$ with the goniometer students of Parnian Girls' Elementary School in Isfahan City, Iran. After screening and initial evaluations involving 422 students, 36 subjects with genu valgum deformity were selected by purposive and convivence sampling method. The study inclusion criteria included being girls aged 9-13 years, the minimal distance of ankle medial malleolus of $2.5 \mathrm{~cm}$, the lack of receiving interventions or exercises that affected the knee posture, no history of visual, auditory, and neuromuscular disorders that affected balance, and no respiratory distress or injury and fractures in the last 6 months. The individuals participated voluntarily in this study. An introductory meeting was held for the selected students' parents on the subject of methods, purposes, and interventions of the research. In total, 30 volunteers were randomly selected and divided into the study groups of test and control ( $\mathrm{n}=15$ /group). The $\mathrm{Q}$ angle measurements were performed using a goniometer with a measuring accuracy of 0.1 . Moreover, we considered the distance of ankle medial malleolus by the coulis with an accuracy of $1.1 \mathrm{~mm}$.

To measure the Q angle, first, the subject stands without flexion, extension, or extra contraction in the lower limbs and as the knees are facing. Then, the center of the goniometer is placed on the center of the patella where its fixed armrests on the tibial tuberosity and movable arm are located along the femur and on the Anterior Supinator Iliac Spine (ASIS). The measured angle shown is the $\mathrm{Q}$ angle (Figure 1).

To measure the distance of the ankle medial malleolus, the barefoot subject stood up straight and without muscle contraction while the patella bones were facing

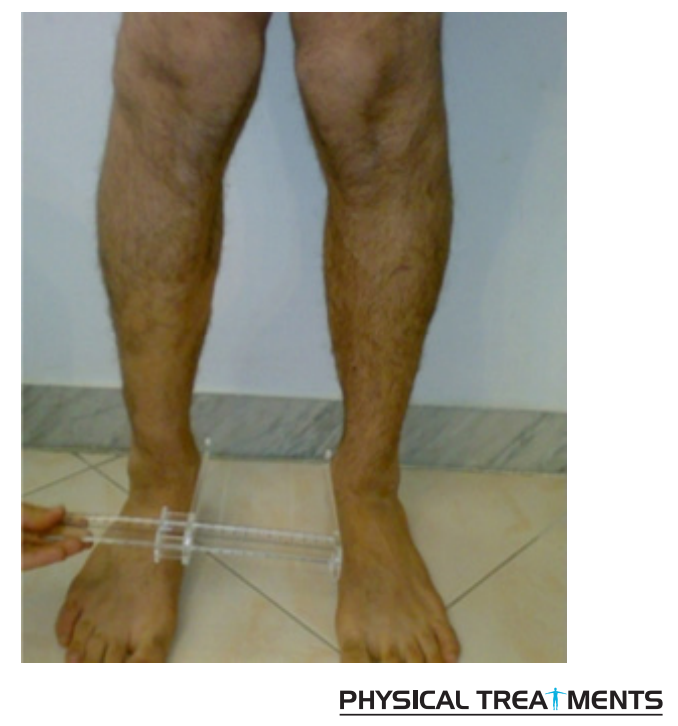

Figure 2. Measuring the distance of ankle medial malleolus by the coulis 
each other. By placing a coulis between the ankle medial malleolus, the distance was recorded (Figure 2).

The experimental group performed exercises for 8 weeks, 3 sessions per week, and about 45-60 minutes per session (5-10 minutes warm-up included movements, such as speed walking, jogging, etc., followed by stretching exercises all over the body, especially the lower limbs, such as hip, knee, and ankle stretch; 40 minutes of TheraBand training; 10 minutes of cooling down lower limbs stretching exercises). However, the control group received no training during this period. The experimental group performed lower limb muscle strengthening exercises on the hip, knees, and ankles (Appendix 1). The training protocol is presented in the Table 1 and 2 [19].

\section{Results}

The demographic characteristics of the study participants are presented in Table 3.

The statistical indicators related to the Q angle and the distance of ankle medial malleolus of the study groups are listed in Table 4

According to Table 5, the covariance test assumptions were met; therefore, a covariance analysis test was used to analyze the collected data.

As per Table 6, ANCOVA data revealed a significant difference between the study groups $(\mathrm{P}<0.05)$. Based on the mean scores, TheraBand exercises significantly impacted the $\mathrm{Q}$ angle and the distance of ankle medial malleolus in the explored subjects.

\section{Discussion}

The present study results suggested that TheraBand exercises significantly reduced the $\mathrm{Q}$ angle and the distance of the ankle medial malleolus in the investigated individuals with genu valgum deformity.

Several factors, including the alignment of the lower limbs, such as the femur, shin, and pelvic muscle strength affected $\mathrm{Q}$ angle in the study participants. A factor influencing the $\mathrm{Q}$ angle is the alignment of the femur. The internal rotation of the femur due to weakness of the hip external rotator muscles increases the valgus in the knee joint and creates malalignment in the lower limbs [20]. Accordingly, this condition could increase the Q angle [21]. Previous studies also revealed that the strength of the abductor and external rotator muscles could significantly affect the normal alignment of the hip, knee, and ankles as well as the Q angle [22]. Increasing the Q angle pulls the patella to the lateral, stretching the quadriceps muscle on the medial side of the knee, and causes muscle imbalance. Biomechanically, the Q angle could change an individual's movement pattern, especially in the foot, leading to internal rotation of the knee and the generation of the genu valgum [22]. Muscle imbalance in a person can lead to the formation of this deformity [23, 24]. Mazidi et al. (2011) investigated the effects of therapeutic exercises on the Q angle among 60 athletes with patellofemoral pain syndrome [25]. They reported that quadriceps strengthening exercises effectively reduced the $\mathrm{Q}$ angle and pain in the studied samples. Delshad et al. (2011) also reported that strengthening training could prevent muscle disability [17].

In this study, the $\mathrm{Q}$ angle was significantly reduced in the studied subjects after 8 weeks of intervention. Prop-

Table 1. The training protocol

\begin{tabular}{|c|c|c|c|c|}
\hline Exercises & Weeks 1 \& 2 (R) & Weeks 3 \& 4 (R) & Weeks 5 \& $6(R)$ & Weeks $7 \& 8(\mathrm{R})$ \\
\hline Squat with foot together & $3 \times 8$ & $3 \times 10$ & $3 \times 15$ & $3 \times 18$ \\
\hline Knee flexion with tibia adduction (stance) & $3 \times 8$ & $3 \times 10$ & $3 \times 15$ & $3 \times 18$ \\
\hline Hip abduction (bird dog) & $3 \times 8$ & $3 \times 10$ & $3 \times 15$ & $3 \times 18$ \\
\hline Knee flexion and extension with supination (prone) & $3 \times 8$ & $3 \times 10$ & $3 \times 15$ & $3 \times 18$ \\
\hline Hip abduction (cross-leg) & $3 \times 8$ & $3 \times 10$ & $3 \times 15$ & $3 \times 18$ \\
\hline Knee flexion with tibia adduction (prone) & $3 \times 8$ & $3 \times 10$ & $3 \times 15$ & $3 \times 18$ \\
\hline Jugging & $3 \times 8$ & $3 \times 10$ & $3 \times 15$ & $3 \times 18$ \\
\hline Hip abduction (lie on side) & $3 \times 8$ & $3 \times 10$ & $3 \times 15$ & $3 \times 18$ \\
\hline
\end{tabular}


Table 2. The training program with thera band

\begin{tabular}{cccc}
\hline Week 1 \& 2 & Week $3 \& 4$ & Week 5 \& 6 & Week 7 \& 8 \\
\hline Yellow & Red & Red & Blue \\
\hline
\end{tabular}

Table 3. Demographic characteristics of the study subjects $(n=15)$

\begin{tabular}{|c|c|c|c|}
\hline \multirow{2}{*}{ Variable } & \multicolumn{2}{|c|}{ Mean \pm SD } & \multirow{2}{*}{$\mathbf{P}$} \\
\hline & Experimental Group & Control Group & \\
\hline Age (y) & $10.76 \pm 1.19$ & $11.1 \pm 1.17$ & 0.3 \\
\hline High (cm) & $148 \pm 2.24$ & $149.19 \pm 2.18$ & 0.17 \\
\hline Weight (kg) & $56 \pm 2.84$ & $57.36 \pm 2.24$ & 0.19 \\
\hline
\end{tabular}

erly causes of reduced Q angle can mention increased strength in the muscles around the knee and the muscles of the femoral abductor. In this regard, Ismaili et al. (2009) stated that factors, such as genu valgum, the weakness of the muscles around the knee, and the external rotation of the tibia increase the Q angle [26].
An exercise used in this study was the strengthening of the femoral abductor muscles. Strengthening this muscle in the hip joint causes the femur to be rotated to external and prevents it from excessive internal rotation. A factor that increases the $\mathrm{Q}$ angle is femoral adduction; thus, it could reduce the $\mathrm{Q}$ angle by increasing the strength of the hip abductor muscles. Furthermore, increasing

Table 4. Statistical indexes of the study groups

\begin{tabular}{|c|c|c|c|c|c|}
\hline Variable & Group & Trial & Mean $\pm S D$ & Depended Samples t-test & $\mathbf{P}$ \\
\hline \multirow{4}{*}{ Q angle (deg) } & \multirow{2}{*}{ Experimental } & Pre-test & $25.1 \pm 2.81$ & \multirow{2}{*}{29.1} & \multirow{2}{*}{0.001} \\
\hline & & Post-test & $18.9 \pm 2.66$ & & \\
\hline & \multirow{2}{*}{ Control } & Pre-test & $25.6 \pm 3.46$ & \multirow{2}{*}{2.2} & \multirow{2}{*}{0.12} \\
\hline & & Post-test & $25.8 \pm 3.32$ & & \\
\hline \multirow{4}{*}{$\begin{array}{l}\text { Distance of ankle malleolus } \\
\qquad(\mathrm{cm})\end{array}$} & \multirow{2}{*}{ Experimental } & Pre-test & $6.2 \pm 1.32$ & \multirow{2}{*}{8.2} & \multirow{2}{*}{0.04} \\
\hline & & Post-test & $2.9 \pm 0.66$ & & \\
\hline & \multirow{2}{*}{ Control } & Pre-test & $5.1 \pm 1.1$ & \multirow{2}{*}{2.2} & \multirow{2}{*}{0.13} \\
\hline & & Post-test & $5.9 \pm 0.66$ & & \\
\hline
\end{tabular}

PHYSICAL TREA $\pitchfork$ MENTS

Table 5. Covariance test assumptions

\begin{tabular}{|c|c|c|c|c|c|c|c|c|}
\hline \multirow{2}{*}{ Variable } & \multirow{2}{*}{ Group } & \multirow{2}{*}{ Trial } & \multicolumn{2}{|c|}{ Shapiro-Wilk test } & \multicolumn{2}{|c|}{ Levene's test } & \multicolumn{2}{|c|}{ Regression } \\
\hline & & & $\mathbf{F}$ & $\mathbf{P}$ & $\mathbf{F}$ & $\mathbf{P}$ & $\mathbf{F}$ & $\mathbf{P}$ \\
\hline \multirow{6}{*}{ Q Angle } & & Pre-test & 0.65 & 0.18 & \multirow{6}{*}{0.396} & \multirow{6}{*}{0.476} & \multirow{6}{*}{0.427} & \multirow{6}{*}{0.24} \\
\hline & Experimental & & 071 & & & & & \\
\hline & & & & & & & & \\
\hline & \multirow{3}{*}{ Control } & Pre-test & 0.67 & 0.9 & & & & \\
\hline & & & & & & & & \\
\hline & & Post-test & 0.74 & 0.27 & & & & \\
\hline \multirow{7}{*}{$\begin{array}{l}\text { The distance of ankle medial } \\
\text { malleolus }\end{array}$} & & Pre-test & 0.76 & 0.29 & \multirow{7}{*}{0.429} & \multirow{7}{*}{0.417} & \multirow{7}{*}{0.512} & \multirow{7}{*}{0.43} \\
\hline & Experimental & & & & & & & \\
\hline & & Post-test & 0.8 & 0.1 & & & & \\
\hline & \multirow{4}{*}{ Control } & & & & & & & \\
\hline & & Pre-test & 0.74 & 0.14 & & & & \\
\hline & & & & & & & & \\
\hline & & Post-test & 0.79 & 0.12 & & & & \\
\hline
\end{tabular}


Table 6. ANCOVA results concerning the effects of the training intervention

\begin{tabular}{|c|c|c|c|c|c|c|}
\hline Variable & Source & Some of Squares & Ds & Mean Squares & $\mathbf{F}$ & $\mathbf{P}$ \\
\hline \multirow{5}{*}{ Q angle } & Corrected model & 16746.1 & 2 & 8.109 & 14.603 & 0.012 \\
\hline & Intercept & 6.84 & 1 & 4.478 & 7.409 & 0.002 \\
\hline & & & & & & \\
\hline & Pre-test score of $Q$ angle & 19.21 & 1 & 9.254 & 16.326 & 0.004 \\
\hline & Group & 7.204 & 2 & 5.105 & 17.258 & 0.003 \\
\hline \multirow{4}{*}{$\begin{array}{l}\text { The distance of ankle medial } \\
\text { malleolus }\end{array}$} & Corrected model & 17256.07 & 2 & 7.009 & 15.712 & 0.003 \\
\hline & Intercept & 4.39 & 1 & 2.499 & 6.625 & 0.01 \\
\hline & Pre-test score of the distance of ankle malleolus & 15.74 & 1 & 10.85 & 19.208 & 0.004 \\
\hline & Group & 6.617 & 1 & 4.231 & 20.039 & 0.002 \\
\hline
\end{tabular}

the strength of the quadriceps muscle stabilizes the patella; therefore, another possible reason for the decrease in Q angle was the increase in quadriceps muscle strength. Sadeghi et al. (2012) examined the effects of corrective exercises on lower extremity deformities in female students [27]. They concluded that a period of strengthening exercises using free weights and stretching exercises to correct the abnormalities of the lower limbs of female students improved the deformities of their lower limbs, including the genu valgum. Moreover, Ashouri et al. (2018) explored the effects of corrective exercises on genu valgum deformity. They argued that 8 weeks of corrective exercises improved the knee and ankle alignment in female high school students [28].

Lee et al. (2016) also indicated that 12 weeks of corrective exercises significantly impacted genu valgum deformity and the proper alignment of the lower limbs [29]. Ghasemi et al. (2017) also reported that the TheraBand strengthening program provided a significant effect on girls' genu varum deformity and has improved the alignment of their hip, knee, and ankles due to the weakening of weakened muscles [5]. Park et al. (2017) revealed that TheraBand resistance training improved knee deformity and reduced the distance between the knees in this group [15]. Haddadnejad et al. (2011) examined the relationship between genu valgum deformity and lower limb strength in adolescent males [30]. The relevant results demonstrated that the genu valgum deformity group had a weaker performance, compared to the genu varum and healthy knee groups. Besides, the genu varum group had a weaker performance, compared to the healthy knee group. They stated that joint muscles play a greater role in performing various jumping tests; thus, these differences in function could be attributed to these muscles and the biomechanical changes in the joints, due to the disruption of the proper alignment of the joints.
The reasons for the increased distance between the ankle's malleolus and the Q angle in the control group was the incorrect habits of these students over time. Besides, the sensitivity of the age of the amulets was important in this regard, because the bones are more formable during the age of growth.

Considering the effects of TheraBand exercises on lower limb muscle strength and the significance of quadriceps and hamstring muscles in the alignment of femur and tibia, the possible etiologies for the distance between the two ankles malleolus could be corrected by strengthening the lower limb muscles. Individuals with a genu valgum deformity encounter weakness in the muscles of the femoral abductor and internal hamstrings, including the semitendinosus and semimembranosus muscles; the exercises used in this protocol were strengthening training for these muscles. The weakness of the adductor muscles and external rotator leads to hip adduction, as well as internal hamstring muscle weakness. Subsequently, this condition causes tibial abduction, followed by the removal of the internal ankles of the ankles. The benefits of TheraBand exercises include variable resistance; these exercises enhance muscle strength and endurance in weakened lower limb muscles. As the strength in the hip abductor muscles increases, the rate of internal rotation and adduction in the hip decreases.

Furthermore, as the strength in the internal hamstring muscles increases, the external rotation of the tibia bone decreases and rotates to the medial side. As a result, it reduces the distance between the medial ankles. Additionally, when the alignment of the femur and tibia is corrected after exercise, the knees close together are somewhat apart, and the medial ankles also become closer. 
The study limitations included goniometer measurement error; the faulty habits of the study subjects, e.g. walking or sitting incorrectly, and the effect of psychological factors on the evaluated subjects' performance.

\section{Conclusion}

The present study data suggested that performing 8 weeks of TheraBand exercises demonstrated a significant improvement in both research variables. Comparing the two research groups, it could be concluded that the experimental group improved due to strengthening exercises with TheraBand. Besides, by increasing strength in weakened muscles, the alignment of the femur and tibia improved and reduced the Q angle and approached the ankle medial malleolus.

Considering the results obtained during 8 weeks of the training intervention, therapists and trainers in sports environments could use strength training exercises to reduce the $\mathrm{Q}$ angle and the distance of ankle medial malleolus in individuals with cruciate knee deformity.

\section{Ethical Considerations}

\section{Compliance with ethical guidelines}

All ethical principles were considered in this article. The participants were informed about the purpose of the research and its implementation stages; they were also assured about the confidentiality of their information; moreover, they were allowed to leave the study whenever they wish, and if desired, the results of the research would be available to them. After describing testprotocol to all subjects before participating in the study, they all signed an informed consent form.

\section{Funding}

This study was approved by the Ethics Committee of the University of Isfahan (Khorasgan) Branch, Islamic Azad University, Department of Sports Injury and Corrective Exercises.

\section{Authors' contributions}

All authors contributed equally in preparing all parts of the research.

\section{Conflict of interest}

The authors declare no conflicts of interest related to this article.

\section{Acknowledgments}

The authors would like to thank the officials of the Faculty of Physical Education at the University of Khorasgan for their valuable cooperation and all students participated in this study.

\section{References}

[1] Mozafaripour E, Rajabi R, Minoonejad H. Anatomical alignment of lower extremity in subjects with genu valgum and genu varum deformities. Physical Treatments. 2018; 8(1):2736. http:/ / dx.doi.org/10.32598/ptj.8.1.27

[2] Shahidi-zandi Z, Amir-seyfaddini, MR, Amiri-Khorasani MT. [Evaluation of lower extremity kinematic characteristics during single-leg landing from different heights in patients with knee valgus deformity (Persian)]. Scientific Journal of Rehabilitation Medicine. 2017; 6(1):122-31. [DOI:10.22037/ JRM.2017.1100299]

[3] Daneshmandi H, Alizadeh MH, Gharakhanlou R. [Corrective exercises (Persian)]. $2^{\text {nd }}$ ed. Tehran: Samat; 2013. http:// opac.nlai.ir/opac-prod/bibliographic/3311312

[4] Daneshmandi H, Alizadeh MH, Gharakhanlou R. [Corrective exercises (Persian)]. $4^{\text {nd }}$ ed. Tehran: Samat; 2006. http:/ / opac.nlai.ir/opac-prod/bibliographic/721627

[5] Ghasemi GA, Sheibani N, Ghaderiyan M. [The effect of 12 weeks of theraband training on the knee position, $\mathrm{Q}$ angle and postural control in persons with genu varum (Persian)]. Journal of Practical Studies of Biosciences in Sport. 2017; 5(10):19-33. [DOI:10.22077/JPSBS.2018.751]

[6] Agaja SB. Factors affecting angular deformities of the knees in Nigerian children-Ilorin experience. West African Journal of Medicine. 2001; 20(4):246-50. [PMID]

[7] Alizadeh MH, Gheitasi M. [Basic concepts of corrective movements (Persian)]. Tehran: Sport Sciences Research Institute of Iran; 2012. http://opac.nlai.ir/opac-prod/bibliographic/ 2879470

[8] van der Esch M, Steultjens M, Harlaar J, Wolterbeek N, Knol D, Dekker J. Varus-valgus motion and functional ability in patients with knee osteoarthritis. Annals of the Rheumatic Diseases. 2008; 67(4):471-7. [DOI:10.1136/ard.2007.071258] [PMID]

[9] Brouwer GM, van Tol AV, Bergink AP, Belo JN, Bernsen $\mathrm{RMD}$, Reijman M, et al. Association between valgus and varus alignment and the development and progression of radiographic osteoarthritis of the knee. Arthritis \& Rheumatism. 2007; 56(4):1204-11. [DOI:10.1002/art.22515] [PMID]

[10] Kubo K, Kanehisa H, Kawakami Y, Fukunaga T. Elasticity of tendon structures of the lower limbs in sprinters. Acta Physiologica Scandinavica. 2000; 168(2):327-35. [DOI:10.1046/j.1365-201x.2000.00653.x] [PMID]

[11] Dashti P, Shabani M, Moazami M. [Comparison of the effects of two selected exercises of Theraband and Pilates on the balance and strength of lower limb in elderly women (Persian)]. The Iranian Journal of Obstetrics, Gynecology and Infertility. 2015; 18(153):1-9. [DOI:10.22038/IJOGI.2015.4698] 
[12] Vincent KR, Braith RW, Feldman RA, Magyari PM, Cutler $\mathrm{RB}$, Persin SA, et al. Resistance exercise and physical performance in adults aged 60 to 83. Journal of the American Geriatrics Society. 2002; 50(6):1100-7. [DOI:10.1046/j.15325415.2002.50267.x] [PMID]

[13] Ciolac EG, Garcez-Leme LE, Greve JMD. Resistance exercise intensity progression in older men. International Journal of Sports Medicine. 2010; 31(6):433-8 [DOI:10.1055/s-0030-1249087] [PMID]

[14] Nobuyuki M, Motohiko M, Izumi T, Noriko S, Tomohiro H, Takeyuki N. Relationship between muscle strength and anthropometric, body composition parameters in Japanese adolescents. Health. 2012; 4(1):1-6. [DOI:10.4236/ health.2012.41001]

[15] Park SR, Ro HL, Namkoong S. The effect of stretching and elastic band exercises knee space distance and plantar pressure distribution during walking in young individuals with genu varum. Journal of The Korean Society of Physical Medicine. 2017; 12(1):83-91. [DOI:10.13066/kspm.2017.12.1.83]

[16] Lovell DI, Cuneo R, Gass GC. The effect of strength training and short-term detraining on maximum force and the rate of force development of older men. European Journal of Applied Physiology. 2010; 109(3):429-35. [DOI:10.1007/ s00421-010-1375-0] [PMID]

[17] Delshad M, Ebrahim K, Gholami M, Ghanbarian A. [The effect of resistance training on prevention of sarcopenia in women over 50 (Persian)]. Journal of Sport Biosciences. 2011; 3(8):123-39. https://jsb.ut.ac.ir/article_23863_en.html

[18] Saki F, Sedaghati P, Aghamohamadi N. [Distal muscle imbalance in girls with and without medial knee displacement during double leg squat (Persian)]. Journal of Sabzevar University of Medical Sciences. 2018; 25(4):537-45. http://jsums. medsab.ac.ir/article_1099_en.html

[19] Pescatello LS. ACSM's guidelines for exercise testing and prescription [AA. Gaeini, A. Samadi, M. Khalesi, Persian trans.]. $9^{\text {th }}$ ed. Tehran: Hatmi; 2014. http://opac.nlai.ir/ opac-prod/bibliographic/3579264

[20] Lee SP, Powers CM. Individuals with diminished hip abductor muscle strength exhibit altered ankle biomechanics and neuromuscular activation during unipedal balance tasks. Gait \& Posture. 2014; 39(3):933-8. [DOI:10.1016/j.gaitt post.2013.12.004] [PMID]

[21] Lin CF, Liu H, Gros MT, Weinhold P, Garrett WE, Yu B. Biomechanical risk factors of non-contact ACL injuries: A stochastic biomechanical modeling study. Journal of Sport and Health Science. 2012; 1(1):36-42. [DOI:10.1016/j. jshs.2012.01.001]

[22] Gribble P, Hertel J, Denegar CR, Buckley WE. The effects of fatigue and chronic ankle instability on dynamic postural control. The Journal of Athletic Training. 2004; 39(4):321-9. [PMID] [PMCID]

[23] Camarata ML, Dhaher YY. Evidence of gender-specific motor templates to resist valgus loading at the knee. Muscle \& Nerve. 2010; 41(5):614-23. [DOI:10.1002/mus.21509] [PMID] [PMCID]

[24] Rajabi R. Laboratory guide to corrective movements for graduate students. Tehran: University of Tehran Publication; 2008 .
[25] Mazidi M, Alizadeh MH, Rajabi R. [Effect of one period of exercise therapy program on $\mathrm{q}$ and popliteal angle in athlethes with pattellofemoral pain syndrome (Persian)]. Journal of Research in Rehabilitation Sciences. 2011: 7(2):206-14. https://www.sid.ir/fa/journal/ViewPaper. aspx?ID $=158057$

[26] Şen M, Çetin S, Ece C, Aydogan A, Çetin HN. Comparison of Quadriceps Q-Angle values of soccer players and wrestlers. Journal of Education and Training Studies. 2019; 7(7):95-101. [DOI:https://doi.org/10.11114/jets.v7i7.4248]

[27] Nikkhouamiri F, Akochakian M, Shirzad Araghi E, Hosein nejad S E. Effect of a course of selected corrective exercises on balance and function of female adolescents with flexible flatfoot. International Journal of Musculoskeletal Pain Prevention. 2019; 4(2):170-9. https://ijmpp.modares.ac.ir/ article-32-36273-en.pdf

[28] Ashouri H. [Evaluation of the effect of eight weeks of corrective movement exercises on cruciate ligament deformity of female high school students in Malayer (Persian)]. $4^{\text {th }} \mathrm{Na}-$ tional Conference on Sports Science and Physical Education of Iran; 2018 May 16, Tehran, Iran. https://civilica.com/ doc/774404/

[29] Lee HS, Kim AR. The Effect of Corrective Exercise in a patient with knee joint valgus deformity: A single-subject AB-A experimental design. Korean Society of Physical Medicine. 2016; 11(1):93-105. [DOI:10.13066/kspm.2016.11.1.93]

[30] Hadadnezhad M, Letafatkar A. [The relationship between genu varum abnormality and lower extremity's performance and strength in teenage footballers (Persian)]. Journal of Research in Rehabilitation Sciences. 2011; 7(2):188-96. https://www.sid.ir/fa/journal/ViewPaper.aspx?ID=158052 


\section{Appendix 1}

Scott's first exercise was called a pair of legs, in which the person stood with the legs straight and, while lowering the center of gravity by flexing the knees, placed the trampoline on the hips, trying to move the hip away and rotate them outward. In this exercise, the upper body is straight and the knees move apart as you move. The second exercise was knee flexion movement with leg adduction. In this movement, the person is standing, sitting on a chair or lying on his stomach with his thighs in line and the bandage on his leg, flexing his knee and at the same time bringing his leg in. Exercise 3 In order to strengthen the abductors and external rotators of the thigh, the person is placed on all fours and while the bandage is on the thigh, he tries to turn the thigh and rotate it externally, alternating with left and right legs. The fourth movement of knee flexion and extension, while the person was lying on his back and trampling the sole of the foot, with 90-degree flexion and knee extension, both leg adduction and ankle supination were performed. The fifth movement of sitting on 4 knees while the bandage was closed on the person's thigh was performed by external rotation and abduction of the thigh, strengthening of the abductors, external rotation of the thigh and stretching of the thigh adapters. The sixth move was a jugging walk, which was performed at the same time with $120^{\circ}$ knee flexion, in order to strengthen the rotators and abductors of the thigh and knee flexors. The seventh movement was hip abduction movement that was performed lying on one side, unilaterally, and in the middle with the left and right legs. In this movement, the theraband was closed on the subject's thigh. 
This Page Intentionally Left Blank 